\title{
A FRAMEWORK FOR UNDERSTANDING THE WORKSPACE ACTIVITY OF DESIGN TEAMS
}

\author{
John C. Tang ${ }^{1,2}$ \\ Larry J. Leifer ${ }^{2}$ \\ ${ }^{1}$ System Sciences Laboratory \\ Xerox Palo Alto Research Center \\ 3333 Coyote Hill Road \\ Palo Alto, California 94304 \\ ${ }^{2}$ Center for Design Research \\ Mechanical Engineering Department \\ Stanford University \\ Stanford, California 94305
}

\begin{abstract}
Small group design sessions were empirically studied to understand better collaborative workspace activity. A conventional view of workspace activity may be characterized as concerned only with storing information and conveying ideas through text and graphics. Empirical evidence shows that this view is deficient in not accounting for how the workspace is used: a) in a group setting, rather than by an individual, and b) as part of a process of constructing artifacts, rather than just a medium for the resulting artifacts themselves. An understanding of workspace activity needs to include the role of gestural activity, and the use of the workspace to develop ideas and mediate interaction. A framework that helps illustrate an expanded view of workspace activity is proposed and supported with empirical data.
\end{abstract}

\section{Introduction}

As part of a study of design activity, we have been examining the workspace activity of collaborative teams. The phrase "workspace activity" broadly refers to any of the actions that occur in the shared space of a team of collaborators working together on a task. For the purposes of this paper, we are examining the workspace in face-to-face settings that include some work surface, such as a whiteboard or sheets of paper. We are focusing on the listing, drawing, and gesturing activities that occur on and around that work surface.

Permission to copy without fee all or part of this material is granted provided that the copies are not made or distributed for direct commercial advantage, the ACM copyright notice and the title of the publication and its date appear, and notice is given that copying is by permission of the Association for Computing Machinery. To copy otherwise, or to republish, requires a fee and/or specific permission.

(C) 1988 ACM 0-89791-282-9/88/0244 \$1.50
Workspace activity has been observed to be a prominent component of design activity, both in our studies of design teams and in previous empirical studies on individuals [Ullman et al., 1987, p. 64; Ballay, 1987, pp. 70-71; Akin, 1978, pp. 75, 80]. Because of its salient role in the design process, there is widespread interest in applying computers and other technologies to support workspace activity, especially in collaborative settings [Stefik et al., 1987; Cook et al., 1987]. Our research is premised on the need to understand the activity that occurs in workspaces in order to inform the design, development, and introduction of these new technologies intended to support it. A better understanding of workspace activity could also lead to insights into improving the design process. 


\begin{tabular}{|c|c|c|c|}
\hline & \multicolumn{3}{|c|}{$\mathrm{ETK}$} \\
\hline Function Action & LIST & DRAW & GESTURE \\
\hline Store information & & & \\
\hline Convey ideas & & & \\
\hline Represent ideas & & & \\
\hline Engage attention & & & \\
\hline
\end{tabular}

Figure 1: Framework for examining workspace activity

In this paper, a framework for examining workspace activity is proposed and supported with empirical data from studies of design activity. This framework lays out relationships between actions that occur in the workspace, and functions that are accomplished through those actions.

The actions describe the means of producing the activity:

List--actions producing non-spatially located text; alpha-numeric notes.

Draw--actions producing graphic objects and marks, including spatially located textual annotations.

Gesture--purposeful body movements which communicate information, such as referring to existing objects in the workspace or enacting simulations.

We are focusing on the list, draw, and gesture activity without accounting much for the talk activity. Similar to a methodological assumption made by Lakin [1986, p. 256], we are studying the accompanying workspace activity in its own right. However, in order to make sense of the workspace activity, this analysis relies heavily on the context of the complete activity of the session (as recorded on videotape), including the verbal dialog. Thus, while not extensively accounting for the verbal activity, we recognize that it is a major part of the session activity and make use of it to help analyze the selected workspace activities.

The functions describe the purpose that the activity accomplishes:

Store information--preserving information in some form for future recall, typically after attaining explicit group agreement.

Convey ideas--transmitting ideas to others in some textual, graphic, or gestural form.

Represent ideas--expressing tentative ideas in some tangible form to allow one's self and others to perceive, react to, and build on them.

Engage attention--directing attention to a region in the workspace, typically to refer to an object or to provide a focus of attention.
This set of actions and functions will be more fully described and discussed in the Analysis section. While this set does not completely describe all of the activities that occur in the workspace, it does suggest a beginning framework through which an emerging understanding can be worked out. In fact, many of the insights that we have gained from this research so far have emerged from the difficulty in categorizing workspace activities and accounting for all of the observed activities. The set of categories presented in this paper is targetted for understanding workspace activity in the service of identifying design implications for workspace support tools. Further research could examine other perspectives on workspace activities (e.g. managing the workspace environment, accessing reference materials [Ballay, 1987 , p. $74,78-80]$ ), as well as workspace activities that occur in other settings (e.g. pairs using a whiteboard [Suchman and Trigg, forthcoming], remote collaboration [Bly, 1988]).

\section{Methodology}

We are exploring the issues of workspace activity through studies in the domain of collaborative design activity. Although we are investigating conceptual design processes, we expect that this research is relevant to collaborative work processes in general. We are studying collaborative design activity because it is a common setting for design, and is likely to be distinct from the individual activity that previous empirical studies in design have examined [Ullman et al., 1987; Ballay, 1987; Akin, 1978; Lera, 1983]. As in the tradition of ethnomethodology and conversation analysis (which study similarly unconstrained types of human activity [Heritage, 1984, pp. 234-238]), we are empirically studying naturalistic activity. This approach is especially appropriate as an exploratory starting point to suggest more focused future research.

Eight sessions of teams of 3-4 people working for approximately 1-1/2 hours were videotaped for analysis. In this paper, we are reporting mainly from the analysis of one complete session (Session A), supplemented with data from a 10 minute section of a second session (Session B), and a third complete session (Session $\mathrm{C}$ ). In these sessions, the groups met 
in a conference room seated around a table with a pad of large paper sheets (supplemented in Session A with private notebooks). Each group worked on a different design task, but all of them emphasized the design of the human-machine interface for a "smart" (microprocessor-controlled) consumer appliance (see sample assignment statement in Appendix). The session observed was the first session that the participants worked on the task as a group, in order to try to capture the earlier, more conceptual stage of their design process. All of the subjects were either educators or graduate students from the Mechanical Engineering Design Division of a major university, and thus shared experiences from a common design culture.

As in any empirical study, the observations reported here come from a limited sample of observed activity. Different workspace activity would be expected with a different work surface (e.g. whiteboard, private notebooks), a different kind of task (e.g. poster graphic, architecture), or a different time-scale problem (e.g. multi-year projects). Group size, familiarity, and working style and the group members' design training and experience would also likely influence workspace activity. While it is not within the scope of this study to account for these and other variables, future research could expand upon the framework proposed here to address these issues.

In Session $A$, the analysis included logging and describing every instance of workspace activity that occurred in the session. This analysis was facilitated by the use of the NoteCards hypertext environment [Halasz et al., 1987] to help structure and represent the data. Figure 2 shows a sample section of transcript with annotations for workspace activity and the area of the work surface which displays the artifacts that were constructed at the time. Each instance of workspace activity was categorized according to the functions and actions laid out in the framework. (Six instances of activity were not included in the tabulated data because they did not fit into any of the function categories.) Having completely logged an entire session also allowed for counting the number of activities in each function and action category and collecting statistics and percentages from the data, as tabulated in Figure 4. Although we are not making any precise statistical claims based on this data, it is presented as evidence for general trends and an indicator of issues for further research.

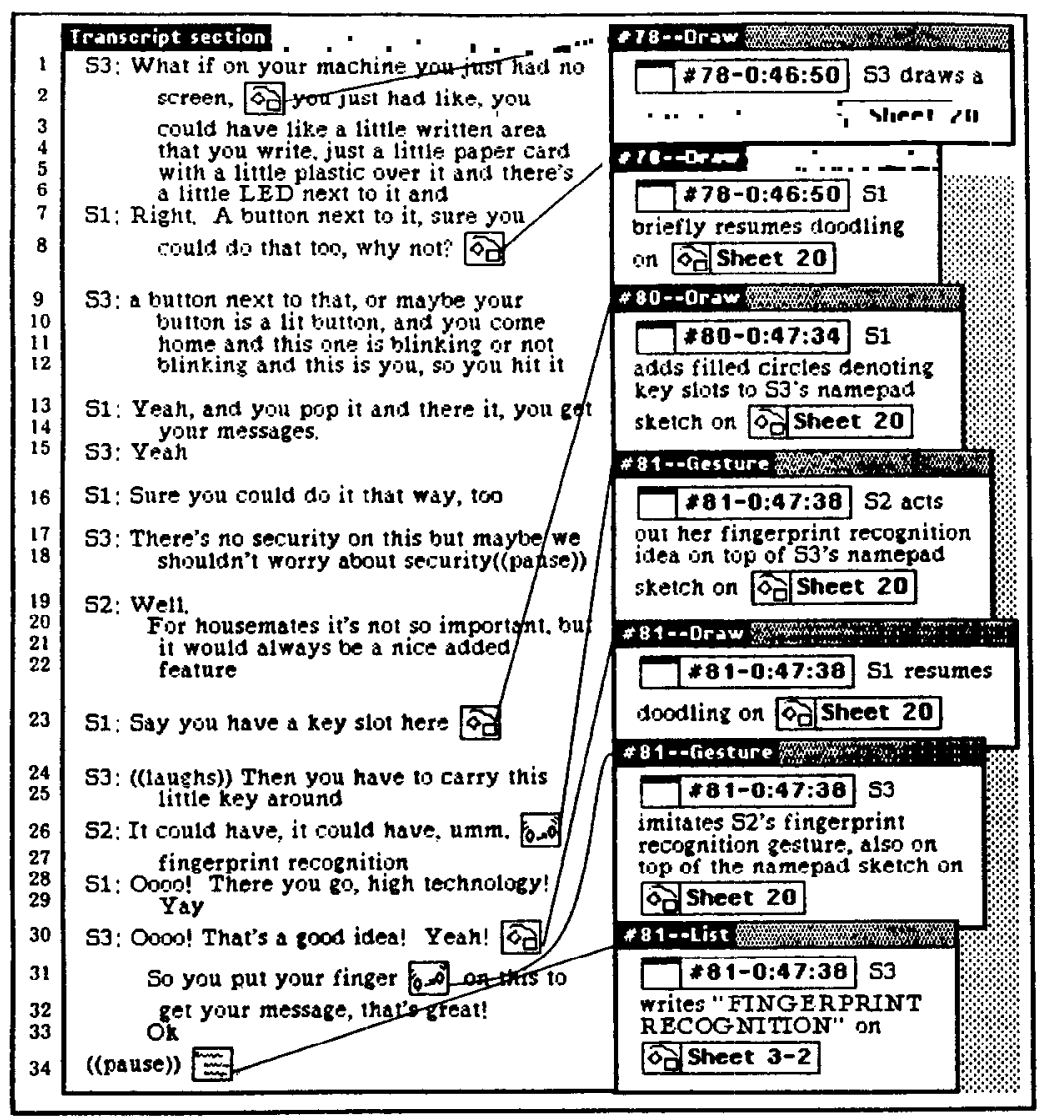

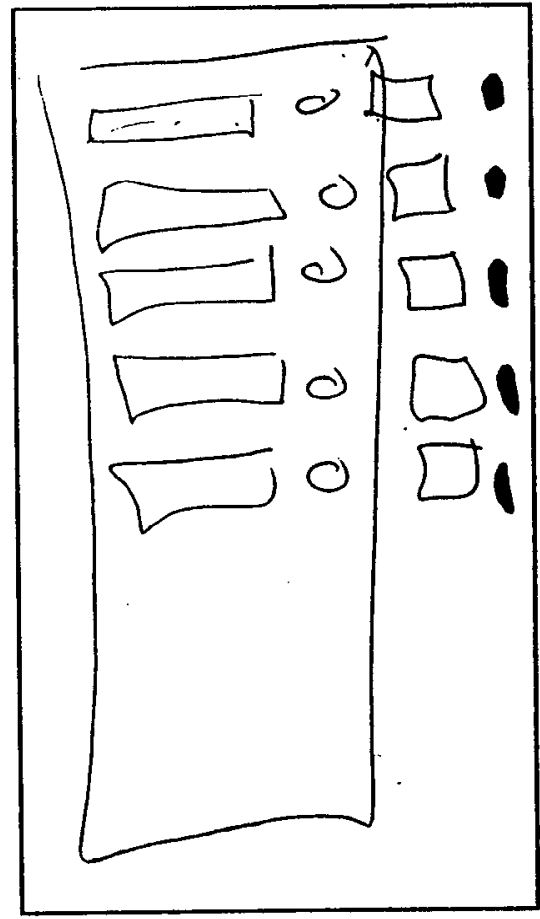

Artifacts created in the workspace

Figure 2: Sample annotated transcript section and workspace artifacts 


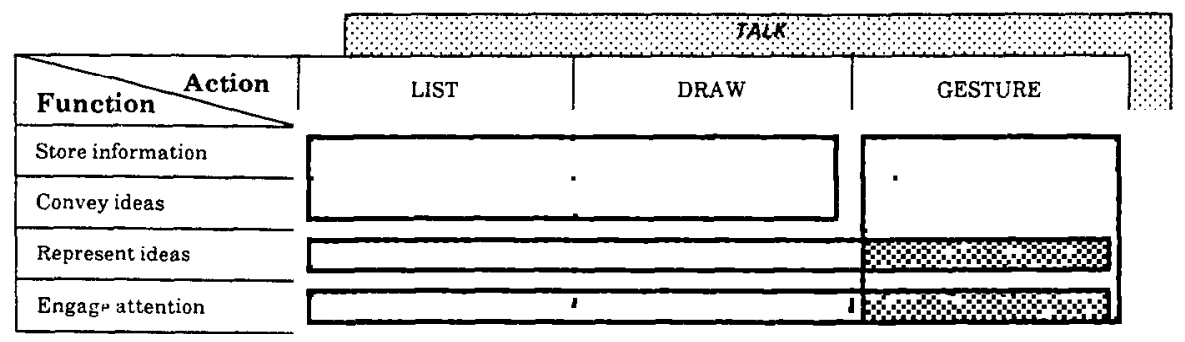

Figure 3: Expanding the Conventional View of Workspace Activity

\section{Analysis}

The proposed framework can be subdivided into four areas: Conventional View, Gestural Expression, Developing Ideas, and Mediating Interaction. This grouping highlights three aspects of workspace activity that go beyond the activities that are conventionally believed to be useful in the workspace. These aspects are discussed and illustrated below with examples from the empirical data.

\section{Conventional View}

As shown in Figure 3, we are labelling as the "conventional view" of workspace activity a characterization of the workspace as primarily a medium for storing information and conveying ideas through listing text and drawing graphics. This subset of functions and actions tends to be the more obvious activities associated with the workspace, and is typically the extent of capability found in conventional Computer Aided Design (CAD) tools. A prototypical example from Session $A$ of using the workspace to store information is shown on line 34 in the sample transcript in Figure 2. There, S3 senses a consensus towards the fingerprint recognition idea (proposed in lines 26-27) and writes it down in her notebook. Lines 1-12 of the sample transcript show an example of S3 conveying an idea as she verbally and graphically expresses her "namepad" proposal.

There were many examples of using text and graphics to store information and convey ideas in our empirical data. However, many of the activities observed could not be classified within the actions and functions of the conventional view. In the numerical statistics collected from session A, just over one-third of all the workspace activities observed could be categorized into this subset of activities (see Figure 4). Thus, the empirical data illustrated the conventional view, but also suggested expanding the framework to include additional aspects of workspace activity.

\section{Gestural Expression}

Gesture is often overlooked as a workspace activity, yet ideas (especially dynamic ones that express some sequence of actions) are often enacted in order to convey them effectively to others. An example is noted in line 26 of the sample transcript when S2 acts out the fingerprint recognition idea.

Although gestures might not typically be thought of as a medium for storing information (because they do not leave behind any permanent record) we have evidence that information can be effectively chunked and remembered through gestures, especially if the gesture is imitated by others and labelled in text or graphics. This aspect of gesture was observed in an idea in Session $\mathrm{C}$ that was adopted by the group, but not readily apparent in the text and graphics records. Reviewing the videotape showed that there was generous acting out of this idea. Through imitating the gesture and labelling it with the phrase "slide and tap", the idea was preserved by the group.

Gestures were also used to refer to existing objects in the workspace, as is described in the Mediating Interaction section. In the Session A statistics in Figure 4, gesture actions constituted over one-third of all the workspace activities logged. One observed characteristic about gestural action is that it is almost always directly accompanied by verbal talk, which is different from the more indirect relationship that listing and drawing can have with the talk.

\begin{tabular}{|c|c|c|c|c|}
\hline Function Action & LIST & DRAW & GESTURE & Total \\
\hline Store information & 40 & 19 & 1 & $60(27 \%)$ \\
\hline Convey ideas & 0 & $22 \quad: 81$ (36\%) & 24 & $46(20 \%)$ \\
\hline Represent ideas & 2 & 41 & 9 & $52(23 \%)$ \\
\hline Engage attention & 0 & 21 & 46 & $67(30 \%)$ \\
\hline Tota! & $42(19 \%)$ & $103(46 \%)$ & $80(35 \%)$ & 225 \\
\hline
\end{tabular}

Figure 4: Frequency of activities of Session A according to functions and actions 


\section{Developing Ideas}

In addition to helping store information and convey ideas, the workspace also plays an active role in the process of developing ideas. Supporting this idea development process is problematic, as evidenced by de:signers' frustrations in using conventional CAD tools to develop ideas fluently as they evolve from free-hand conceptual sketches to formal specifications. This aspect of developing ideas involves the use of the workspace as part of an interactive process, rather than only as a medium for the resulting text-graphic artifacts.

We observed 2 key features that the designers utilized in developing ideas: a) being able to readily try out representations of ideas in the public workspace, and b) having those representations gradually evolve into distinct artifacts, often through other participants building on and modifying them. An example is shown in the transcript sample in Figure 2. On line 2, S3 draws a representation of her "namepad" idea into the workspace. S1 builds on it by adding keyhole slots in line 23 , and S2 gestures over it when she enacts the fingerprint recognition idea in line 26. This illustrates how, in less than 1 minute, each participant was able to represent a different thought in the workspace and build on those artifacts previously created by others to develop the namepad idea. We have several similar episodes of activity where this fluent expression of ideas in the workspace and the ability to interact and build on representations created by others contributes to the process of developing ideas.

A similar example that was observed in another session highlights an additional workspace capability used in developing ideas. In Session B, one participant started drawing privately, producing a graphical object. Other participants took notice of this object and began working on it, and it ultimately evolved in to the representation that recorded the gist of the session. The migration of this object from a private drawing to a public representation illustrates the dual private/public nature of the workspace: individuals feel that they can begin representing an idea privately in some portion of the workspace, but ultimately everyone has a shared view of the workspace, so that an idea can be gradually made public as it matures. We need to understand how to incorporate these capabilities into computer tools so that they become facile and fluent enough to support this process of developing ideas.

\section{Mediating Interaction}

The workspace plays a key role in mediating the interaction of the collaborators, primarily as a vehicle to engage people's attention. Besides directing attention to an existing object in the workspace (by referring, retracing, or otherwise emphasizing it), one can occupy one's own attention (e.g. by doodling or working privately), or draw attention to one's self in preparation for enacting a gesture. These activities also help mediate the conversational turn-taking. By coordinating some workspace action with the talk, one can take a turn, indicate the desire for the next turn, or hold onto a turn. For example, in Session A, one participant began to sketch a drawing in a private area of the workspace, demonstrating apparent loss of interest in the discussion and sustaining his own interest by doodling. The others eventually directed their attention to his drawing, and yielded a turn to him, asking him to explain it. Using the workspace in this way to mediate interaction depends on having a shared view on the workspace and a close proximity (either real or virtual, e.g. through video) among the participants and work surface.

These attention engaging activities are usually subtle (and thus unremarkable) and typically do not add any new information by themselves. Yet they should not be ignored, since they play a vital role in sustaining the pace of the interaction. One of the observations noted by Bly [1988] is that the absence of these attention focusing activities in situations that do not afford this workspace capability noticeably detracts from the interaction process. The numerical data in Figure 4 from Session $A$ indicates that these activities constitituted a substantial portion (just under one-third) of the workspace activities. More research is needed to explore ways for tools to support these interactional functions, or at least make sure not to interfere with existing resources and mechanisms for interaction.

\section{Conclusions}

Empirical observation of design activity reveals functions and actions that extend beyond the conventional view of workspace activity. To a large degree, observing these additional aspects of workspace activity stems from studying collaborative activity, rather than individual activity, and examining the process of workspace activity, rather than just the resulting artifacts. Gestural expression is more prominent in collaborative situations, since it is used mainly to demonstrate actions to others and focus the attention of others to specific locations. Group settings also emphasize the process of developing ideas collaboratively, in contrast to a perspective which suggests that each individual generates ideas and then uses the workspace only to convey and store those ideas. Group settings also highlight the prominence of mediating interaction as a substantial aspect of collaborative design activity, and show the workspace to be a key resource for that activity. The work of gesture and some of the other process oriented workspace activities would likely be overlooked if only the resulting workspace artifacts were examined, since these activities do not leave behind any substantial artifacts. 
Recognizing these additional aspects of workspace activity has important implications for the design of workspace support tools. Since gestures often occur in relation to existing objects in the workspace, a sense of proximity between work surface and participants should be maintained. Further research could investigate how to support gestural activity, possibly by including the accompanying verbal activity, since gestures are typically accompanied by talk. A shared view of all the workspace activity and the ability to interact and build upon others' work facilitates the process of developing ideas. Tools should also support fluent expression of ideas and allow smooth development from informal to formal representations. Sharing a common view of the workspace and maintaining a sense of closeness are also important for preserving the subtle cues used to mediate interaction.

\section{Acknowledgements}

We would especially like to thank Sara Bly for the many discussions and insights that she contributed and Lucy Suchman for her consistent supervision. We also thank Scott Minneman for reviewing previous drafts of this paper. We would like to acknowledge Xerox PARC for funding this research, and the members of the Interaction Analysis Lab there for their comments and help in analyzing the videotapes. We would like to acknowledge the Center for Design Research at Stanford University and the System Sciences Laboratory at Xerox PARC (especially the NoteCards Support staff), for the individuals in those groups that have taken an interest in this work, and the environments they provide that has fostered this research.

\section{Appendix}

Assignment statement for Session A:

In teams of 3 or 4 , design a custom multi-function telephone for the user and environment of your choice.

It should have at least three of the following functions: auto-dial and redial, answering machine, calendar and clock, log or diary, call waiting and forwarding, hold and transfer, conferencing, call-back, speaker-phone or any other you might think of relevant to your particular user(s).

The goal of this project is for you to be able to design complex computer-based products which are easy, efficient, safe and satisfying to use. You should be able to use scenarios to describe users and environments, task analysis to determine information needs, key-stroke models to predict efficiency and simple prototypes and story-boards to check learning.

\section{References}

Akin, Ömer, "How do architects design?", Artificial Intelligence and Pattern Recognition in Computer Aided Design, J. Latombe, Ed., IFIP, North-Holland Publishing Company, 1978, pp. 65-103.

Ballay, Joseph M., "An experimental view of the design process", System Design: Behavioral Perspectives on Designers, Tools, and Organizations, William B. Rouse and Kenneth R. Boff, Eds., New York: North-Holland, 1987, pp. 65-82.

Bly, Sara A., "A use of drawing surfaces in different collaborative settings", Second Conference on Computer-Supported Cooperative Work, Portland, OR, September 1988.

Cook, Peter, Clarence Ellis, Mike Graf, Gail Rein, and Tom Smith, "Project Nick: Meetings Augmentation and Analysis", ACM Transactions on Office Information Systems, Vol. 5, No. 2, April 1987, pp. 132-146.

Halasz, Frank G., Thomas P. Moran, and Randall H. Trigg, "NoteCards in a Nutshell", Proceedings of the $A C M C H I+G I$ Conference, Toronto, April 1987, pp. 45-52.

Heritage, John, "Conversation Analysis", Garfinkel and Ethnomethodology, Cambridge: Polity Press, 1984, pp. 233-292.

Lakin, Fred, "A performing medium for working group graphics", Proceedings of the Conference on Computer-Supported Cooperative Work, Austin, TX, December 1986, pp. 255-266.

Lera, Sebastian, "Synopses of some recent published studies of the design process and designer behaviour", Design Studies, Vol. 4, No. 2, April 1983, pp. 133-140.

Stefik, Mark, Gregg Foster, Daniel G. Bobrow, Kenneth Kahn, Stan Lanning, and Lucy Suchman, "Beyond the chalkboard: Computer support for collaboration and problem solving in meetings", Communications of the ACM, Vol. 30 , No. 1, January 1987, pp. 32-47.

Suchman, L. and R. Trigg, "Constructing the behavior of shared conceptual objects: A study of work at the whiteboard", in Situation, Occasion and Context in Activity, J. Lave and S. Chaiklin, Eds., Cambridge, UK: Cambridge University Press, in preparation.

Ullman, David G., Larry A. Stauffer, and Thomas G. Dietterich, "Toward Expert CAD", Computers in Mechanical Engineering, Vol. 6, No. 3, November/December 1987, pp. 56-70. 